\title{
Radiation recall dermatitis: report of two cases
}

\author{
Francisco Acevedo • Paula Arriagada • \\ Carolina Ibañez $\cdot$ Claudia Ortega $\cdot$ Pablo Muñoz \\ Yerko Borghero $\cdot$ Marisa Bustos $\cdot$ Cesar Sanchez
}

Received: 30 November 2012/Accepted: 7 March 2013/Published online: 6 April 2013

(C) The Japan Society of Clinical Oncology 2013

\begin{abstract}
Systemic treatment for cancer including chemotherapy or biologic agents can cause different changes in the skin or mucous membranes. These changes may represent a known adverse reaction to these drugs, although other etiologies should be considered. One of these, radiation recall dermatitis, is a rare and unpredictable phenomenon. Herein, we report two cases of this condition in women with metastatic breast cancer secondary to gemcitabine and the combination of capecitabine and ixabepilone.
\end{abstract}

Keywords Recall dermatitis · Breast cancer · Ixabepilone · Capecitabine $\cdot$ Gemcitabine

\section{Introduction}

Radiation recall dermatitis (RRD) is a rare inflammatory reaction occurring in a previously irradiated area, precipitated by the administration of certain drugs. It is importance to note the difference between the radiosensitization phenomenon with inflammatory changes present during treatment with radiotherapy, versus the recall phenomenon that occurs as a late, unpredictable event. Because of its

F. Acevedo $(\bowtie) \cdot$ P. Arriagada $\cdot$ C. Ibañez $\cdot$ P. Muñoz

Y. Borghero - M. Bustos - C. Sanchez

Department of Hematology-Oncology, Faculty of Medicine,

Pontificia Universidad Catolica de Chile, Diagonal Paraguay

319, Santiago, Chile

e-mail: fnacevedo@gmail.com

C. Ortega

Department of Radiology, Faculty of Medicine, Pontificia

Universidad Catolica de Chile, Santiago, Chile rareness and non-specific symptoms, it poses a great diagnostic challenge that requires a high index of suspicion. We describe two cases of RRD secondary to gemcitabine and the combination of capecitabine and ixabepilone, and a short review of literature on the topic.

\section{Case report}

\section{Case 1}

A 34-year-old woman was diagnosed with metastatic triple negative breast cancer. Her bone scan showed multiple bone lesions and computed tomography (CT) scan showed no visceral disease. She received a 5-fluorouracil $(600$ $\left.\mathrm{mg} / \mathrm{m}^{2}\right)$, doxorubicin $\left(60 \mathrm{mg} / \mathrm{m}^{2}\right)$ and cyclophosphamide $\left(600 \mathrm{mg} / \mathrm{m}^{2}\right)$-based chemotherapy for six cycles and then a cleaning mastectomy was performed. After recovery from surgery, paclitaxel plus bisphosphonates were initiated with no toxicity reported.

Six months later, she developed local and systemic progression with subcutaneous nodular lesions in the mastectomy scar and liver metastasis. A skin biopsy was performed which showed diffuse infiltration of tumor cells. She underwent hyperfractionated radiation therapy, using a $6 \mathrm{MV} \mathrm{X}$-ray, to the chest wall at doses of 57, 8 Gray (Gy) in two-daily fractions delivered over 20 days (Fig. 1). The treatment was well-tolerated with only acute erythema followed by signs of re-epithelialization.

Two weeks later, she was offered a new chemotherapy regimen based on capecitabine (at doses of $1250 \mathrm{mg} / \mathrm{m}^{2}$ twice daily) plus ixabepilone $\left(40 \mathrm{mg} / \mathrm{m}^{2}\right)$. Three days after completion of chemotherapy she presented to the emergency room with intense pain over the recently irradiated 

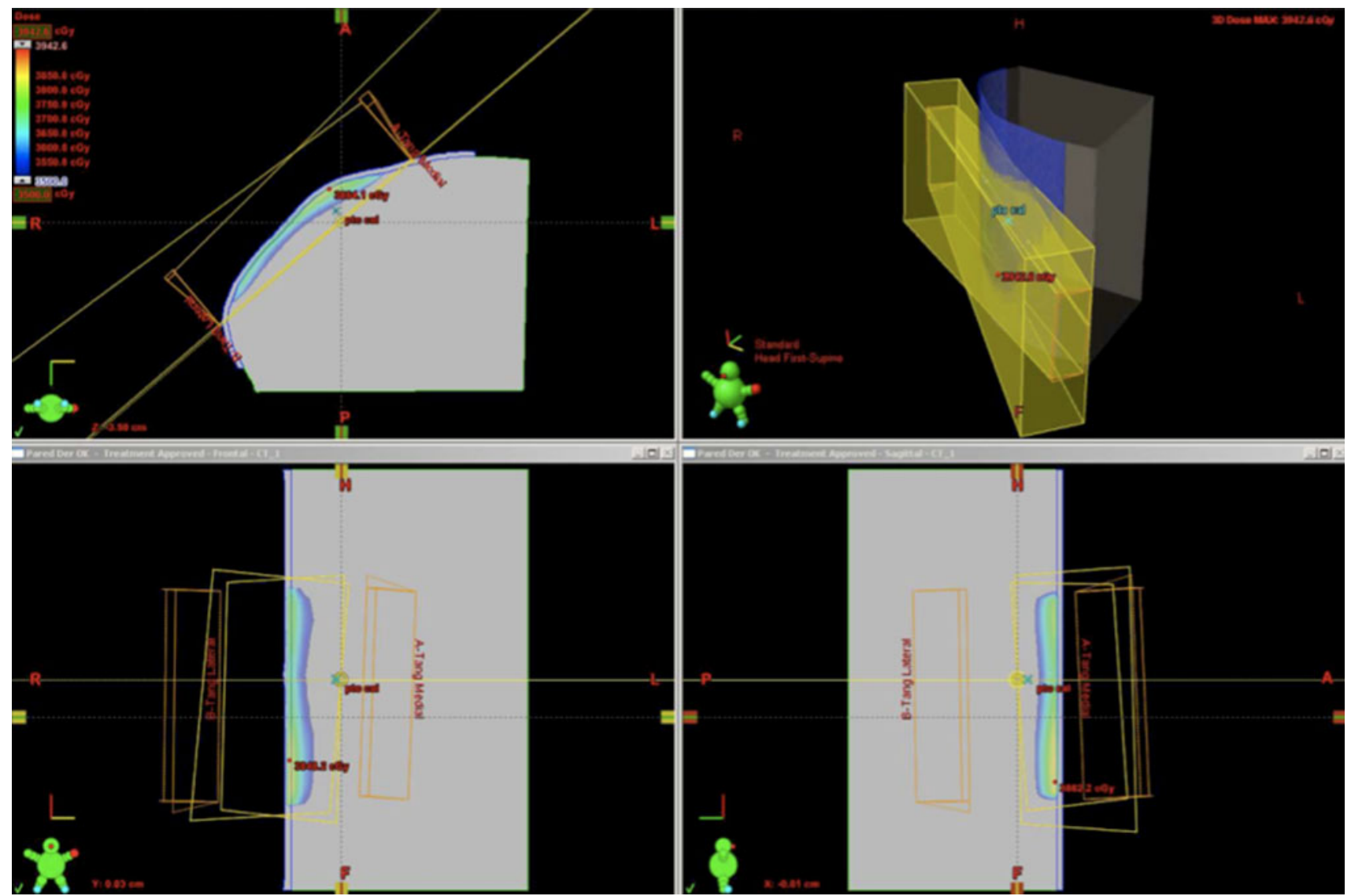

Fig. 1 Radiation planning diagram shows area to be irradiated in a patient with metastatic breast cancer
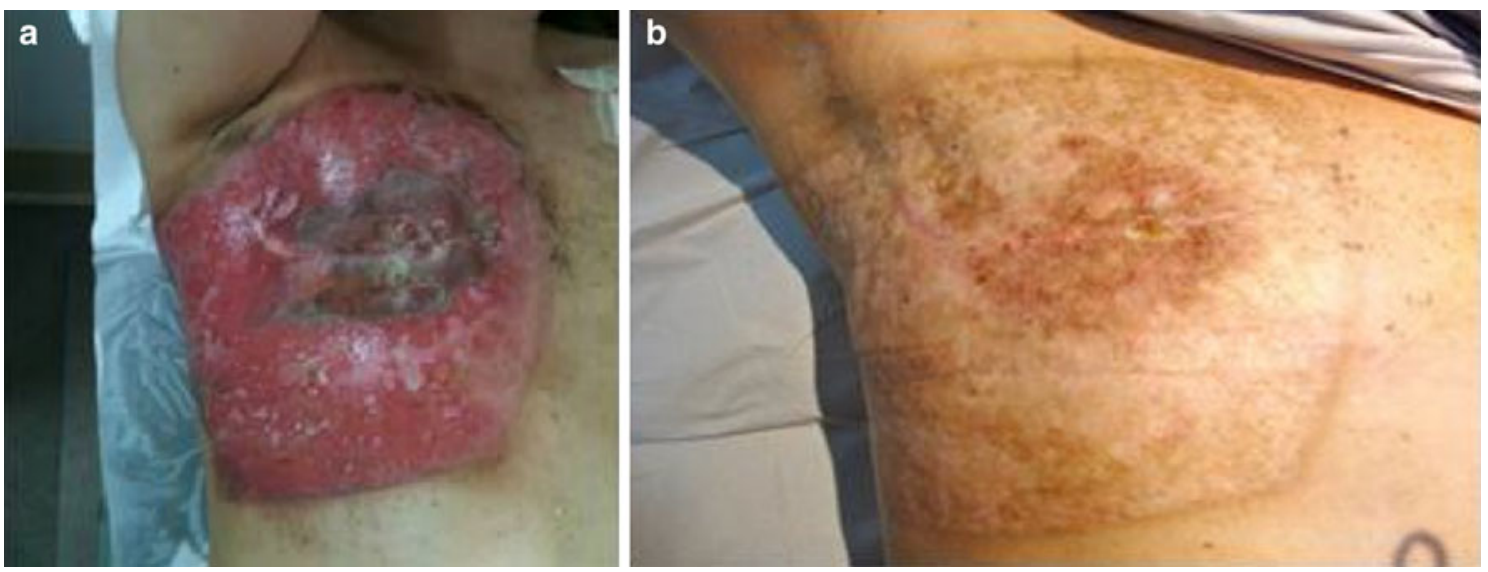

Fig. 2 a Photography showing a well-demarcated zone with intense erythema and vesicles in a previously irradiated area. b The same patient, 2 weeks after suspension of chemotherapy and local treatment

chest wall. Physical examination revealed a well-demarcated rectangular area associated with erythema and vesicles (Fig. 2a). The lesions were consistent with the clinical diagnosis of RRD. Chemotherapy was suspended and local treatment with isotonic saline and moisturizing lotion produced an excellent response (Fig. 2b).
Case 2

A 54-year-old woman diagnosed with T1N0, ER positive, HER-2 positive breast cancer was prescribed treatment consisting of a partial mastectomy plus radiation therapy and adjuvant chemotherapy with doxorubicin and 

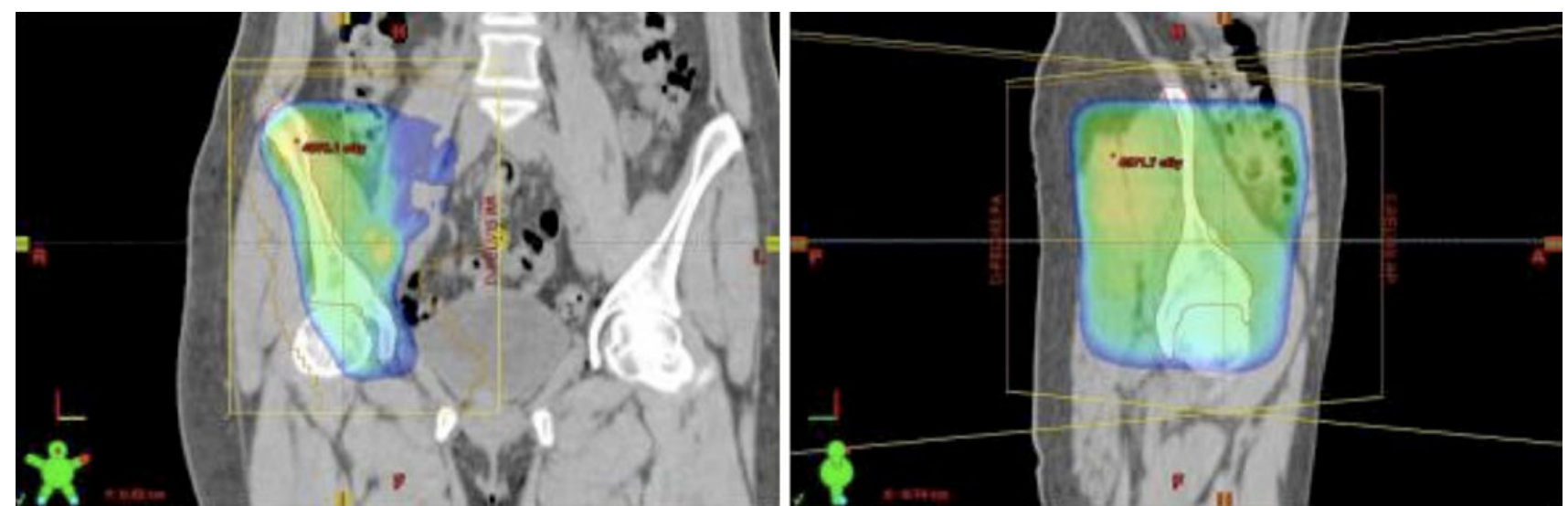

Fig. 3 Dose distribution obtained from simulation CT scan in a patient with metastatic breast cancer

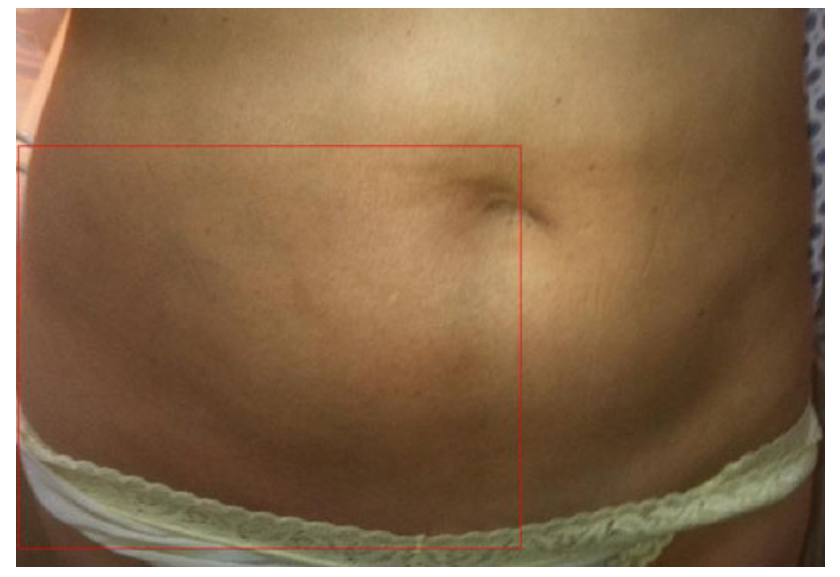

Fig. 4 Photography showing abdominal wall muscle and slight overlying erythema that matched the radiation field, consistent with the diagnosis of radiation recall dermatitis

cyclophosphamide, followed by trastuzumab for 1 year and tamoxifen to complete 5 years. Two years after diagnosis, asymptomatic lung metastasis was documented. She was then offered docetaxel, carboplatin and trastuzumab-based chemotherapy and achieved a stable disease status for almost a year. After multiple progressions and different lines of chemo and hormone therapy she developed symptomatic right-sided sacroiliac joint and ischial bone metastases. She received palliative-intended radiation (39 Gy in 13 fractions with 18 MV photons) with good clinical response and no reports of skin reaction (Fig. 3). A new chemotherapy regimen based on gemcitabine $1200 \mathrm{mg}$ and trastuzumab $100 \mathrm{mg}$ every 3 weeks, in addition to letrozole and bisphosphonate was started. Five months after the initiation of the new chemotherapy, she referred intense abdominal pain in the right lower quadrant associated with slight erythema, warmth and tenderness of the overlying skin that matched the radiation field (Fig. 4). CT scan showed diffuse swelling of the right abdominal wall muscles and the corresponding soft tissue. Signs of ileocolitis were also noticed (Fig. 5). Laboratory examination confirms the presence of myositis with elevation of creatine kinase to $1300 \mathrm{U} / \mathrm{L}$. A diagnosis of RRD was made. Treatment with prednisone and NSAID was begun with partial response.

\section{Discussion}

Radiation recall dermatitis (RRD) is a rare and unpredictable phenomenon that can occur weeks, months or years after exposure to radiation. It is characterized by an acute inflammatory reaction, confined to areas previously irradiated, and usually triggered by the administration of certain drugs or sun exposure [1].

Although it is generally associated with the administration of antineoplastic chemotherapy, cases of RRD have been described with the use of other drugs such as quinolones, macrolides, statins or calcium antagonists [2-5]. D'Angio [6] published the first report in 1959, describing that dactinomycin could "reactivate" latent radiation effects in normal tissue. Since then, a series of cases have been reported, but still there are no epidemiological studies that allow us to fully characterize this syndrome. The majority of cases described occurred after exposure to monodrug treatment with anthracyclines, taxanes or antimetabolites, especially gemcitabine and capecitabine [7-14].

Clinically, the majority of cases manifest as an acute cutaneous inflammatory reaction (dermatitis) with erythema, maculopapular or vesicular lesions, and pain followed by dry desquamation in the affected area. However, in the most severe cases might be persistent painful vesicles and necrosis. In one third of cases there is involvement of other structures besides the skin with reports describing cases of pneumonitis, myositis or enteritis [12, 15-17]. 

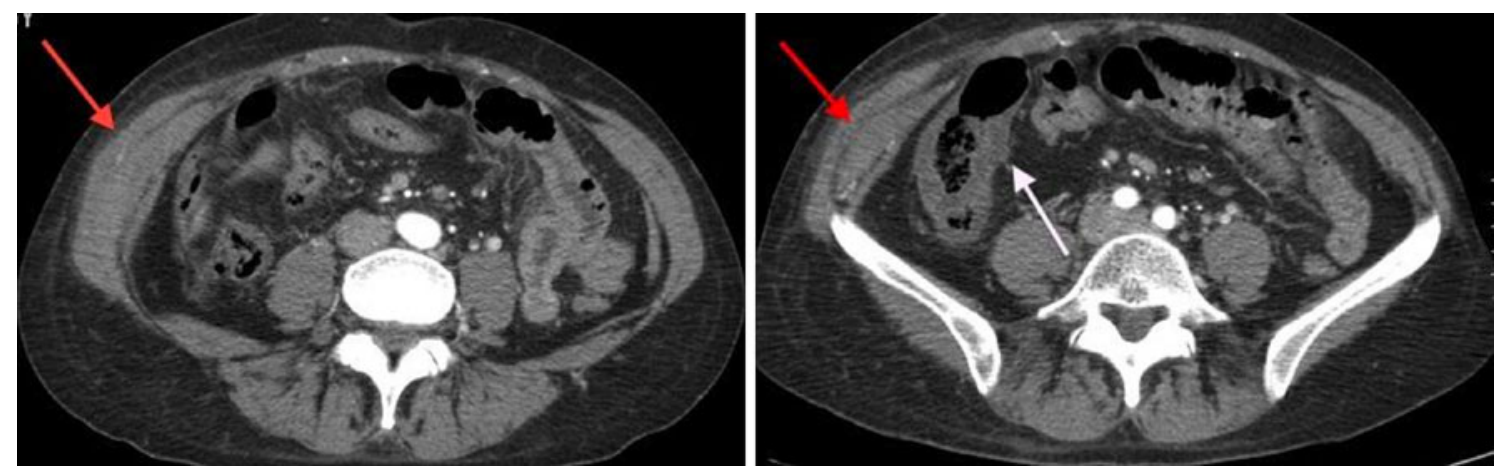

Fig. $5 \mathrm{CT}$ scan of abdomen showing muscle edema and thickening (red arrow). Signs of colitis with submucosal edema on contrast fixation are noticed (white arrow)

Intense acute radiation reactions during treatment with radiotherapy are not considered a risk factor for subsequent RRD [18].

It is important to stress the difference between RRD and radiosensitization. In the latter, the effect of the radiation is enhanced during or few days after treatment with radiation therapy. In RRD the phenomenon can occur many days after the treatment has been completed, without any direct effects of radiation corresponding more to an idiosyncratic reaction [1]. The timing was set in an arbitrary way with some authors, suggesting that skin reactions brought on by drugs given less than 7 days after radiotherapy should be considered radiosensitization [1].

Even though RRD might be considered an infrequent event, being that most of the relevant information is obtained from case reports, Kodym describes its appearance in $8.8 \%$ of 91 patients receiving palliative chemotherapy for metastatic disease [19]. It is also estimated that the probability of being diagnosed with RRD is associated with the time interval between radiation and drug exposure and RRD symptoms. A short interval between the end of radiation therapy and the beginning of chemotherapy is a known risk factor to develop the condition. Nevertheless, there are reports of cases occurring years or even decades later [20].

Based on the fact that many patients with RRD had previously received other drugs known to be also responsible, with no evidence of reaction, the response appears to be drug specific [21]. However, it is unlikely that the cytotoxic properties of chemotherapeutic agents by themselves are responsible for the phenomenon since it has an unpredictable response and in many cases the re-exposure to the drug does not produce symptom recurrence [22]. Several theories have been proposed to explain the pathophysiologic basis for RRD; however none of them has been fully validated so it remains as a poorly understood phenomenon. It has been proposed that RRD corresponds to hypersensitivity idiosyncratic drug reaction and it could be considered analogous to fixed drug eruptions [18]. Thus, local skin reaction would be related to the continued secretion of pro-inflammatory cytokines induced by exposure to radiation and subsequently activation of a nonimmune inflammatory pathway, which decreases the threshold to inflammatory response. In this scenario, the presence of a chemotherapeutic agent would trigger the overexpression of these cytokines producing the phenomenon of RRD [23].

Local vascular damage and radiation-induced depletion of stem-cells, or their inability to proliferate in situations of cytotoxic exposure, have also been hypothesized as potential etiologies of RRD [24, 25].

In the particular case of capecitabine, it has been suggested that the recall dermatitis could be caused by overexpression of the enzyme thymidylate phosphorylase, which induces angiogenesis in the irradiated area and local activation of the pro-drug in this zone [12].

In this paper, we presented two cases that illustrate the most frequent clinical presentations of RRD. The first case illustrates a dermatitis induced by the combination of capecitabine and ixabepilone with full recovery after withdrawal of the drug. Although cases of RRD have been reported with these agents used alone, this is the first case described with their combination $[1,12,26]$. In the second example we report a case of myositis associated with ileocolitis post-administration of gemcitabine and trastuzumab. Here, it is important to note that the patient presented with the classical RRD described with gemcitabine. Unlike other drugs (including trastuzumab) where dermatitis is the major manifestation, the recall reaction with gemcitabine mainly affects muscular tissue and internal organs, such as in this case [13, 27, 28]. Despite the fact that trastuzumab has been reported as a causal agent, we think it unlikely that this biological agent is responsible for $\mathrm{RRD}$, based on the fact that the patient received this drug before without reporting any side effects.

The main part of the treatment involves cessation of the responsible agent. Most cases will resolve with this single action and supportive care. A rechallenge with the same 
drug, using lower but more frequent doses has been successfully reported in the literature [26]. The time needed for complete healing varies with the type of offending drug, with longer times being described for oral agents [25]. Several adjuvant treatments have been described including systemic administration of corticosteroids, NSAIDs or antihistaminics according to the clinical severity of the reaction $[1,15]$. Local treatment with hydrocortisone, silver sulfadiazine or sodium hyaluronate has been also used successfully [26, 29].

Conflict of interest The authors of the Manuscript: "Radiation Recall Dermatitis. Report of two cases" declarethat we have no conflict of interest

\section{References}

1. Burris HA 3rd, Hurtig J (2010) Radiation recall with anticancer agents. Oncologist 15:1227-1237

2. Wernicke AG, Swistel AJ, Parashar B et al (2010) Levofloxacininduced radiation recall dermatitis: a case report and a review of the literature. Clin Breast Cancer 10:404-406

3. Vujovic O (2010) Radiation recall dermatitis with azithromycin. Curr Oncol 17:119-121

4. Abadir R, Liebmann J (1995) Radiation reaction recall following simvastatin therapy: a new observation. Clin Oncol (R Coll Radiol) 7:325-326

5. Taunk NK, Haffty BG, Goyal S (2011) Radiation recall 5 years after whole-breast irradiation for early-stage breast cancer secondary to initiation of rosuvastatin and amlodipine. J Clin Oncol 29:e661-663

6. D'Angio GJ, Farber S, Maddock C (1959) Potentiation of x-ray effects by actinomycin-D. Radiology 73:175-177

7. Haas RL, de Klerk G (2011) An illustrated case of doxorubicininduced radiation recall dermatitis and a review of the literature. Neth J Med 69:72-75

8. Gzell CE, Carroll SL, Suchowerska N et al (2009) Radiation recall dermatitis after pre-sensitization with pegylated liposomal doxorubicin. Cancer Invest 27:397-401

9. Raghavan VT, Bloomer WD, Merkel DE (1993) Taxol and radiation recall dermatitis. Lancet 341:1354

10. Shenkier T, Gelmon K (1994) Paclitaxel and radiation-recall dermatitis. J Clin Oncol 12:439

11. Mizumoto M, Harada H, Asakura $\mathrm{H}$ et al (2006) Frequency and characteristics of docetaxel-induced radiation recall phenomenon. Int J Radiat Oncol Biol Phys 66:1187-1191
12. Saif MW, Black G, Johnson M et al (2006) Radiation recall phenomenon secondary to capecitabine: possible role of thymidine phosphorylase. Cancer Chemother Pharmacol 58:771-775

13. Friedlander PA, Bansal R, Schwartz L et al (2004) Gemcitabinerelated radiation recall preferentially involves internal tissue and organs. Cancer 100:1793-1799

14. Mallik S, Gupta S, Munshi A (2010) Memoirs of differential radiation doses: gemcitabine-induced radiation recall. Acta Oncol 59:261-262

15. Schweitzer VG, Juillard GJ, Bajada CL et al (1995) Radiation recall dermatitis and pneumonitis in a patient treated with paclitaxel. Cancer 76:1069-1072

16. Lock M, Sinclair K, Welch S et al (2011) Radiation recall dermatitis does not suggest the need to discontinue chemotherapy. Oncol Lett 2:85-90

17. Fakih MG (2006) Gemcitabine-induced rectus abdominus radiation recall. JOP 7:306-310

18. Camidge R, Price A (2001) Characterizing the phenomenon of radiation recall dermatitis. Radiother Oncol 59:237-245

19. Kodym E, Kalinska R, Ehringfeld C et al (2005) Frequency of radiation recall dermatitis in adult cancer patients. Onkologie 28:18-21

20. Burdon J, Bell R, Sullivan J et al (1978) Adriamycin-induced radiation recall 15 years after radiotherapy. JAMA 239:931

21. Bokemeyer C, Lampe C, Heneka M et al (1996) Paclitaxelinduced radiation recall dermatitis. Ann Oncol 7:775-776

22. Lampkin BC (1969) Skin reaction to vinblastine. Lancet 1:891

23. Azria D, Magne N, Zouhair A et al (2005) Radiation recall: a well recognized but neglected phenomenon. Cancer Treat Rev $31: 555-570$

24. Bostrom A, Sjolin-Forsberg G, Wilking N et al (1999) Radiation recall: another call with tamoxifen. Acta Oncol 38:955-959

25. Hellman S, Botnick LE (1977) Stem cell depletion: an explanation of the late effects of cytotoxins. Int J Radiat Oncol Biol Phys 2:181-184

26. Ford JN, Newton M, Jordan C et al (2013) Successful rechallenge after ixabepilone-induced radiation recall dermatitis using an alternative dosing strategy. J Oncol Pharm Pract 19:89-92

27. Shrimali RK, McPhail NJ, Correa PD et al (2009) Trastuzumabinduced radiation recall dermatitis. First reported case. Clin Oncol 21:634-635

28. Chung C, Stuart D, Keyes M (2009) Radiation recall reaction induced by adjuvant trastuzumab (herceptin). Case Rep Med 2009:307894

29. Bauer SM, Bauer C (2009) The use of sodium hyaluronate for the treatment of radiation recall dermatitis. J Oncol Pharm Pract 15:123-126 